\title{
Relative Span Weighted Localization of Uncooperative Nodes in Wireless Networks
}

\author{
Christine Laurendeau and Michel Barbeau \\ School of Computer Science, Carleton University \\ 1125 Colonel By Drive, Ottawa, ON Canada K1S 5B6 \\ Tel: 613-520-2600; Fax: 613-520-4334 \\ E-mail: \{claurend,barbeau\}@scs.carleton.ca
}

\begin{abstract}
Increasingly ubiquitous wireless technologies require novel localization techniques to pinpoint the position of an uncooperative node, whether the target be a malicious device engaging in a security exploit or a low-battery handset in the middle of a critical emergency. Such scenarios necessitate that a radio signal source be localized by other network nodes efficiently, using minimal information. We propose two new algorithms for estimating the position of an uncooperative transmitter, based on the received signal strength (RSS) of a single target message at a set of receivers whose coordinates are known. As an extension to the concept of centroid localization, our mechanisms weigh each receiver's coordinates based on the message's relative RSS at that receiver, with respect to the span of RSS values over all receivers. The weights may decrease from the highest RSS receiver either linearly or exponentially. Our simulation results demonstrate that for all but the most sparsely populated wireless networks, our exponentially weighted mechanism localizes a target node within the regulations stipulated for emergency services location accuracy.
\end{abstract}

\section{Introduction}

Given the pervasiveness of cellphones and other wireless devices, compounded with the associated expectation of permanent connectivity, it is perhaps not surprising that the abrupt dashing of such presumptions makes headline news. A recent spate of cases in Canada have highlighted the tragic consequences of failing to locate the source of an emergency 911 cellphone call. These events have spurred the Canadian Radiotelevision Telecommunications Commission (CRTC) to regulate the same wireless Enhanced 911 (E911) provisions [1] as the Federal Communications Commission (FCC) in the U.S. [2] Under Phase II of the FCC and CRTC plans, network-based localization, where other nodes (whether base stations or other handsets within range) estimate the position of a device, must accurately reveal a target location within 100 meters $67 \%$ of the time and within 300 meters in $95 \%$ of cases.

Self-localization achieved with handset-based localization techniques can produce granular results. For example with the Global Positioning System (GPS), a precision of ten meters may be achieved [3]. But self-localization is not feasible in all scenarios. An uncooperative node is one that cannot be relied upon to determine its coordinates, 
for example a defective sensor, a malicious device engaging in a security exploit or a low-battery handset in a critical situation.

In a sufficiently densely populated wireless network, the source location of a given message may be approximated from the coordinates of receiving devices, assuming an omnidirectional propagation pattern. We propose two localization algorithms that estimate a transmitting node's position as the weighted average of receiver coordinates, assuming a single message is received from the target node. We describe a relative span weighted localization (RWL) mechanism, where the concept of weighted moving average is adapted to provide a linear mapping between the weight assigned to a receiver's coordinates and the relative placement of its received signal strength (RSS) value within the overall RSS span. We further propose an exponential variation of RWL, dubbed relative span exponential weighted localization (REWL). This approach is conceptually related to an exponential moving average and relies on an exponential weight correspondence between a receiver's coordinates and its relative situation within the RSS span. We evaluate the RWL and REWL algorithms using simulated RSS reports featuring a variety of node densities, number of receivers, and amount of signal shadowing representative of environment-based RSS fluctuations. We also test our localization mechanisms with RSS values harvested from an outdoor field experiment.

Section 2 provides an overview of existing work in centroid-based localization techniques. Section 3 outlines the centroid localization schemes on which our new algorithms are based. Section 4 describes our linearly and exponentially weighted location estimation mechanisms. Section 5 evaluates the performance of both algorithms using simulated and experimental RSS values. Section 6 concludes the paper.

\section{Related Work}

Centroid localization (CL) has been suggested as an efficient location estimation method that never fails to produce a solution, unlike existing geometric and algebraic localization approaches [4-6]. The original incarnation of CL is described by Bulusu et al. [7], and localizes the transmitting source of a message to the $(x, y)$ coordinates obtained from averaging the coordinates all receiving devices within range. Weighted centroid localization (WCL), as proposed by Blumenthal et al. [8], assigns a weight to each of the receiver coordinates, as inversely proportional to either the known transmitterreceiver (T-R) distance or the link quality indicator available in ZigBee/IEEE 802.15.4 sensor networks [9]. Behnke and Timmermann [10] extend the WCL mechanism for use with normalized values of the link quality indicator. Schuhmann et al. [11] conduct an indoor experiment to determine a set of fixed parameters for an exponential inverse relation between T-R distances and the corresponding weights used with WCL. Orooji and Abolhassani [12] suggest a T-R distance-weighted averaged coordinates scheme, where each receiver's coordinates are inversely weighted according to its distance from the transmitter. But this approach assumes that the receivers are closely co-located and that the T-R distance to at least one of the receivers is known a priori. 


\section{Centroid Localization}

We outline the centroid localization approaches on which our novel algorithms are based, and introduce the notation used throughout the description of our mechanisms.

Notation. The estimated coordinates of the transmitter we are striving to locate are denoted as $\widehat{p}=(\widehat{x}, \widehat{y})$. Each receiver $R_{i}$ is situated at a point of known coordinates $p_{i}=\left(x_{i}, y_{i}\right)$. For the sake of simplicity in our algorithm descriptions, we depict operations on receiver points $p_{i}$. In fact, two separate calculations occur. The approximated $\widehat{x}$ coordinate is computed from all the receiver $x_{i}$ coordinates, and $\widehat{y}$ is calculated from the $y_{i}$ coordinates.

Given a set of known points $p_{i}$ in a Euclidian space, for example a number of receivers within radio range of a target transmitter to be localized, Bulusu et al. [7] approximate the location $\widehat{p}$ of a node from the centroid of the known points $p_{i}$ as follows:

$$
\widehat{p}=(1 / n) \times \sum_{i=1}^{n} p_{i}
$$

where $n$ represents the number of points.

In the simple CL approach, all points are assumed to be equally near the target node. Blumenthal et al. [8] argue that some points are more likely than others to be close to target node. Their WCL scheme aims to improve localization accuracy by assigning greater weight to those points which are estimated to be closer to the target and less weight to the farther points. The weighted centroid is thus computed as:

$$
\widehat{p}=\sum_{i=1}^{n}\left(w_{i} \times p_{i}\right) / \sum_{i=1}^{n} w_{i}
$$

with $w_{i}=\left(d_{i}\right)^{-g}$ where $d_{i}$ is the known distance between the target node and point $p_{i}$, and the exponent $g$ influences the degree to which remote points participate in estimating the target location $\widehat{p}$. Values of $g$ are determined manually, with Blumenthal et al. and Schuhmann et al. [11] promoting different optimal values, depending on the experimental setting.

\section{Relative Span Weighted Localization}

Assuming an uncooperative node, we cannot presume to know a priori the set of T$\mathrm{R}$ distances $d_{i}$ or the optimal value of $g$ in a given outdoor environment. Further, we cannot estimate values of $d_{i}$ from the log-normal shadowing model, as the transmitter effective isotropic radiated power (EIRP) may not be known. We therefore introduce the concept of relative span weighted localization in order to estimate the location of a transmitter with minimal information available at a set of receivers. Our approach adapts the concept of moving average from a weighting method over time and applies it to WCL in the space domain. But rather than ascribing weights according to known or approximated T-R distances, we weigh each receiver coordinates according to the relative placement of its RSS value within the span of all RSS reports for a given transmitted message. We assign greater weight to the receiver coordinates whose RSS value 
is closer to the maximum of the RSS span and thus closer to the transmitter. Conversely, lesser weight is ascribed to receivers with lower RSS values, as they are deemed farther from the transmitter. The receiver coordinates may be weighted linearly or exponentially.

Definition 1 Minimal/Maximal RSS. Let $\mathbb{R}$ be the set of all receivers within range of a given message $\mathcal{M}_{T}$ originating from an uncooperative transmitter $T$. Let $\Upsilon$ denote the set of RSS values measured at each receiver $R_{i} \in \mathbb{R}$ for message $\mathcal{M}_{T}$, such that: $\Upsilon=\left\{v_{i}: v_{i}\right.$ is the RSS value for message $\mathcal{M}_{T}$ at $R_{i}$ for all $\left.R_{i} \in \mathbb{R}\right\}$

Then we define the minimal and maximal $R S S$ values, $\mathcal{V}_{\text {min }}$ and $\mathcal{V}_{\text {max }}$, for message $\mathcal{M}_{T}$, as the smallest and largest RSS values in $\Upsilon$, such that: $\mathcal{V}_{\text {min }}=\min \left\{v_{i} \in \Upsilon\right\}$

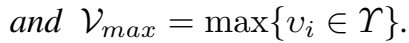

Definition 2 RSS Span. Let the minimal and maximal RSS values for a message $\mathcal{M}_{T}$ be as stated in Definition 1. We define the RSS span $\mathcal{V}^{\Delta}$ for this message at a set of receivers $\mathbb{R}$ as the maximal range in RSS values over all receivers, such that: $\mathcal{V}^{\Delta}=$ $\mathcal{V}_{\text {max }}-\mathcal{V}_{\text {min }}$.

We describe two relative span weighted localization algorithms, both computing a weighted centroid as defined in Equation (2), but with novel approaches for computing the weights assigned to each receiver coordinates.

\subsection{Linearly Weighted Localization}

The RWL algorithm computes a centroid of receiver coordinates, each weighted linearly according to the relative position of the receiver's RSS value within the RSS span.

Algorithm 1 RWL Algorithm. The relative span weighted localization (RWL) algorithm estimates a transmitter's coordinates $\widehat{p}$ as the weighted centroid of all receiver coordinates $p_{i}$, as defined for WCL in Equation (2), but with a linearly increasing weight assigned to each receiver according to its presumed proximity to the transmitter. Given the RSS values in $\Upsilon$, as found in Definition 1, and the RSS span $\mathcal{V}^{\Delta}$ determined according to Definition 2, the weight $w_{i}$ of each receiver $R_{i}$ is computed from the relative placement of its RSS value $v_{i}$ in the RSS span, as $w_{i}=\left(v_{i}-\mathcal{V}_{\text {min }}\right) / \mathcal{V}^{\Delta}$, for each $R_{i} \in \mathbb{R}$

The relative span weighted centroid thus becomes:

$$
\widehat{p}=\frac{\sum_{i=1}^{n}\left[\left(v_{i}-\mathcal{V}_{\text {min }}\right) \times p_{i}\right]}{\sum_{i=1}^{n}\left(v_{i}-\mathcal{V}_{\text {min }}\right)} \quad \text { where } n=|\mathbb{R}|
$$

\subsection{Exponentially Weighted Localization}

Exponentially weighted moving averages (EMAs) have been used for a variety of forecasting applications, for example in Muir [13], to predict future values based on past 
observations, with more weight exponentially ascribed to more recent data. A weighting factor $\lambda$ is used as a parameter to control the proportion of weight assigned to recent observations with respect to past ones.

We adapt the EMA concept, as described by Roberts [14], from rating observations over time for the purpose of weighting receiver coordinates over the space domain. While EMA favors more recent observations in time with a weighting factor of $\lambda$, we bolster receivers that are likely to be closer to a transmitter and thus feature higher RSS values. In addition, rather than increasing the weighting factor exponent by one for each observation in time, we correlate the exponent with the relative position of each receiver's RSS value within the RSS span.

Algorithm $2 \boldsymbol{R E W L}$ Algorithm. The relative span exponentially weighted localization (REWL) algorithm estimates a transmitter's coordinates $\widehat{p}$ as the weighted centroid of all receiver coordinates $p_{i}$, as defined for WCL in Equation (2), but with exponential weight assigned to each receiver according to a weighting factor $\lambda$. Given the RSS values in $\Upsilon$ as found in Definition 1, the weight $w_{i}$ of each receiver $R_{i}$ is computed from the relative placement of its $R S S$ value $v_{i}$ in the RSS span, as $w_{i}=(1-\lambda)^{\left(\mathcal{V}_{\max }-v_{i}\right)}$, for each $R_{i} \in \mathbb{R}$.

The relative span exponentially weighted centroid thus becomes:

$$
\widehat{p}=\frac{\sum_{i=1}^{n}\left[(1-\lambda)^{\left(\mathcal{V}_{\max }-v_{i}\right)} \times p_{i}\right]}{\sum_{i=1}^{n}(1-\lambda)^{\left(\mathcal{V}_{\max }-v_{i}\right)}} \quad \text { where } n=|\mathbb{R}|
$$

\section{Performance Evaluation}

We evaluate the performance of the RWL and REWL algorithms using simulated RSS values and experimental ones harvested from an outdoor field experiment.

\subsection{Simulation Results}

We ran the RWL and REWL mechanisms on simulations featuring a variety of node densities and number of receivers. For each of 10000 executions, we generate a random transmitter position within a $1000 \times 1000 \mathrm{~m}^{2}$ simulation grid. We define our node densities as the number of nodes per $100 \times 100 \mathrm{~m}^{2}$. For every node density $d \in$ $\{0.25,0.50,0.75,1.00,2.00,3.00,4.00,5.00,6.00,7.00,8.00,9.00,10.00\}$, we position $d$ nodes per $100 \times 100 \mathrm{~m}^{2}$ in uniformly distributed positions on our simulation grid. For each node, we compute a RSS value based on the log-normal shadowing model [15], with a random amount of signal shadowing generated along a log-normal probability distribution. We assume two different radio propagation environments with path loss constants obtained from outdoor experiments. For the $2.4 \mathrm{GHz} W i F i / 802.11 \mathrm{~g}$ frequency, we use propagation values measured by Liechty et al. $[16,17]$, where a signal shadowing standard deviation is measured at nearly $\sigma=6 \mathrm{dBm}$. For the $5.8 \mathrm{GHz}$ frequency, licensed for vehicular networks [18], we make use of the constants determined 
by Durgin et al. [19], with a signal shadowing standard deviation close to $\sigma=8 \mathrm{dBm}$. Similar experiments by Schwengler and Gilbert corroborate the amount of signal shadowing commonly experienced at this frequency [20]. Our setup allows us to gauge the performance of relative span weighted localization based on propagation environments featuring different amounts of signal fluctuations. Once our simulated nodes are positioned, we determine which ones can be used as receivers. We set all receiver sensitivity to $-90 \mathrm{dBm}$, and the nodes that feature a RSS value above the sensitivity are deemed within range of the transmitter and thus become receivers. The non-receiver nodes are subsequently ignored as out of range.

For each execution, we use the known coordinates of all receivers to compute a possible position for the transmitter, according to four algorithms: the maximum RSS receiver method, where a transmitter is assumed to be at exactly the receiver position with the highest RSS value; the CL approach, as set out by Bulusu et al. in Equation (1); the RWL algorithm using Equation (3); and the REWL algorithm as set forth in Equation (4), given three different values for the weighting factor $\lambda \in\{0.10,0.15,0.20\}$. We assess the performance of each mechanism according to its location accuracy, computed as the Euclidian distance between the estimated position $\widehat{p}$ and the actual transmitter location, averaged over all executions. Our results are deemed accurate within \pm 3 meters in a $95 \%$ confidence interval.

Figure 1 plots the average location error for each tested algorithm, given all defined node densities, for frequency $2.4 \mathrm{GHz}$. The corresponding results at the $5.8 \mathrm{GHz}$ frequency may be found in [21]. We find that while higher densities consistently yield greater location accuracy, a larger amount of signal shadowing results in higher location errors. For example, for all densities, the REWL algorithm, with the $2.4 \mathrm{GHz}$ frequency and $\sigma=6 \mathrm{dBm}$, yields a location error consistently less than 75 meters, while the same mechanism at the $5.8 \mathrm{GHz}$ frequency and $\sigma=8 \mathrm{dBm}$ reaches an error of 105 meters. In general, we find an error increase of roughly $50 \%$ for every $2 \mathrm{dBm}$ of additional signal shadowing standard deviation. For both frequencies and all node densities, the REWL algorithm with weighting factor of $15 \%(\lambda=0.15)$ achieves optimal results.

We assessed the performance of each algorithm, and in particular the REWL $(\lambda=$ 0.15) mechanism, when compared to the E911 regulations for location accuracy. While every method evaluated meets the E911 requirements at $2.4 \mathrm{GHz}$ with moderate signal shadowing ( $\sigma=6 \mathrm{dBm})$, none of the mechanisms succeed with $5.8 \mathrm{GHz}$ and a larger amount of shadowing $(\sigma=8 \mathrm{dBm})$ [21]. However, even in the latter case, the REWL approach is nearly adequate. Given the smaller amount of signal shadowing found at 2.4 $\mathrm{GHz}$, the REWL $(\lambda=0.15)$ algorithm meets the E911 location accuracy requirements for every node density, as seen in Figure 2. For larger amounts of shadowing at 5.8 $\mathrm{GHz}$, only the smallest node density of 0.25 per $100 \times 100 \mathrm{~m}^{2}$ fails to meet the E911 standard, as shown in Figure 3. Even in a heavily shadowed environment, higher node densities can accurately localize a transmitter within 100 meters $67 \%$ of the time and within 300 meters in $95 \%$ of cases.

Orooji et al. [12] simulate a cluster of seven cells, each featuring a base station with a one kilometer radius, in order to compute the location of a mobile station. A very small amount of signal shadowing $\sigma \in\{1,2\} \mathrm{dBm}$ is taken into account. Even though their proposed T-R distance-weighted method assumes a known distance to one of the 


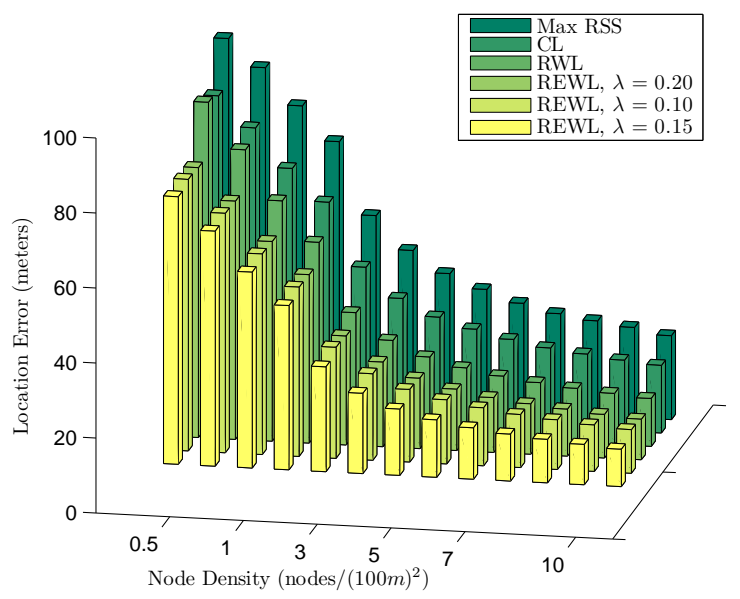

Fig. 1. Algorithm Location Error by Node Density for $2.4 \mathrm{GHz}$

base stations, the mean location error is 48 meters, with $95 \%$ of executions resulting in a location error less than 103 meters. Our RWL and REWL $(\lambda=0.15)$ algorithms for 2.4 $\mathrm{GHz}$ with eight receivers yield an average 37 and 34 meter location error respectively. RWL locates a transmitter within 100 meters $98 \%$ of the time, while REWL does so in $99 \%$ of cases. Thus over a similarly sized simulation grid, our RWL and REWL mechanisms consistently yield more accurate results.

\subsection{Experimental Results}

We conducted an outdoor field experiment with four desktop receivers statically arranged in the corners of a rectangular area $80 \times 110 \mathrm{~m}^{2}$ in size. Each receiver collected the RSS values of packets transmitted by a laptop from each of ten separate locations. Only the messages simultaneously received by the four desktops were retained. The localization algorithms were executed on each message, and the location error for each algorithm averaged over all transmitter locations can be found in Table 1. We find that the RWL and REWL mechanisms perform far better than the maximum RSS receiver and CL approaches, with a gain in location accuracy of up to $40 \%$. On average, the RWL, REWL with $\lambda=0.15$, and REWL with $\lambda=0.20$ mechanisms perform equally well, with no algorithm emerging as clearly superior to the others. This may be due to our small experimental data set (approximately 400 messages), when compared to simulation results obtained over 10000 executions. While our simulations also found consistently similar results between the RWL and REWL mechanisms, the larger amount of simulated data allows us to draw more fine-tuned conclusions. 


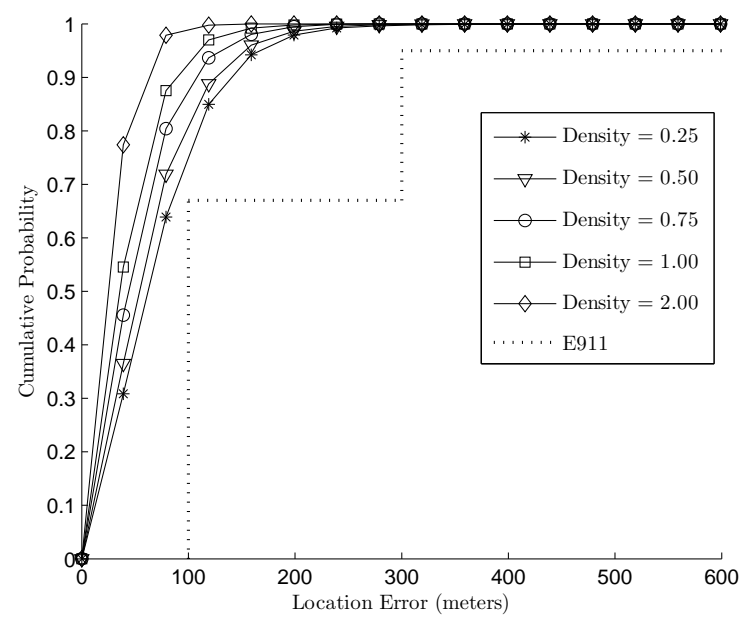

Fig. 2. REWL Location Error CDF by Node Density for $2.4 \mathrm{GHz}$

Table 1. Average Location Error for All Experimental Transmitter Locations

\begin{tabular}{|c|c|c|c|c|c|c|}
\hline \multirow{2}{*}{ Algorithm } & \multirow{2}{*}{ Max RSS } & \multirow{2}{*}{$\mathrm{CL}$} & \multirow{2}{*}{ RWL } & \multicolumn{3}{|c|}{ REWL } \\
\cline { 4 - 7 } & & & & $\lambda=0.10$ & $\lambda=0.15$ & $\lambda=0.20$ \\
\hline \hline Average Location Error (meters) & 40 & 46 & 28 & 33 & 29 & 28 \\
\hline
\end{tabular}

\section{Conclusion}

We propose a wireless network-based localization mechanism for estimating the position of an uncooperative transmitting device, whether it be a malfunctioning sensor, an attacker engaging in a security exploit or a low-battery cellphone in a critical emergency. We extend the concept of weighted centroid localization and describe two additional receiver coordinate weighting mechanisms, one linear and the other exponential, that assume no knowledge of the T-R distances nor of the transmitter EIRP. We adapt the concept of moving averages based on observations over time to the space domain. We ascribe linear and exponential weights to each receiver coordinates, based on the relative positioning of the receiver's RSS value within the RSS span of all receivers.

We tested our relative span weighted localization algorithms with simulated and experimental RSS values, using two frequencies featuring different amounts of signal shadowing. We found that our algorithms yield lower location errors than the existing centroid localization method. As expected, the location accuracy increases as more nodes participate in the localization effort. For example with REWL $(\lambda=0.15)$ at 2.4 $\mathrm{GHz}$, one node per $100 \times 100 \mathrm{~m}^{2}$ localizes a transmitter within 44 meters, while ten nodes per $100 \times 100 \mathrm{~m}^{2}$ do so in less than ten meters. Yet the location accuracy decreases as the amount of signal shadowing between different receivers increases, with an average decrease of approximately $50 \%$ for every $2 \mathrm{dBm}$ of additional signal shad- 


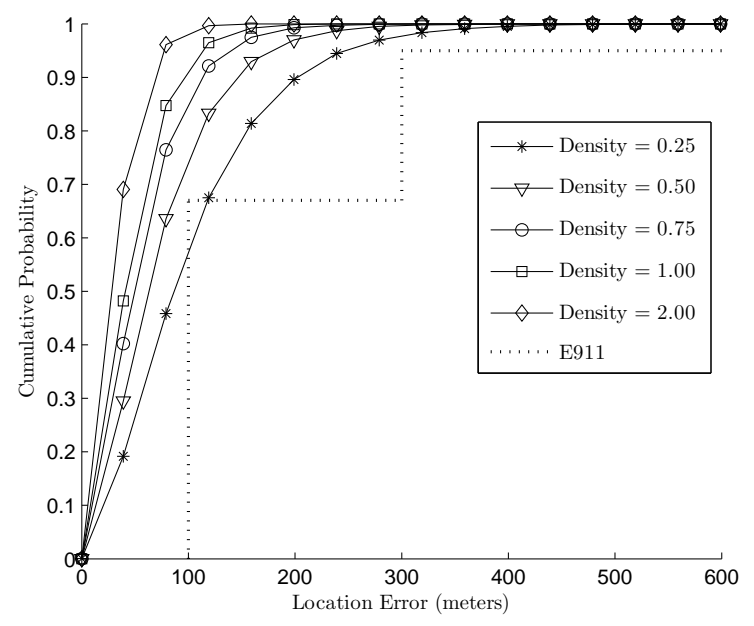

Fig. 3. REWL Location Error CDF by Node Density for $5.8 \mathrm{GHz}$

owing standard deviation. We conclude that the exponential variation of our relative span weighted localization algorithm achieves a location accuracy that meets the FCC regulations for Enhanced 911, for all densities with moderate amounts of signal shadowing and for all but the smallest node densities with extensive shadowing.

\section{Acknowledgment}

The authors gratefully acknowledge the financial support received for this research from the Natural Sciences and Engineering Research Council of Canada (NSERC), and the Automobile of the 21st Century (AUTO21) and Mathematics of Information Technology and Complex Systems (MITACS) Networks of Centers of Excellence (NCEs).

\section{References}

1. Canadian Radio-television Telecommunications Commission: Implementation of Wireless Phase II E9-1-1 Service. Telecom Regulatory Policy CRTC 2009-40 (February 2009)

2. Federal Communications Commission: 911 Service. FCC Code of Federal Regulations, Title 47, Part 20, Section 20.18 (October 2007)

3. Nielson, J., Keefer, J., McCullough, B.: SAASM: Rockwell Collins' Next Generation GPS Receiver Design. In: Proceedings of the IEEE Position Location and Navigation Symposium. (March 2000) 98-105

4. Liu, C., Wu, K., He, T.: Sensor Localization with Ring Overlapping Based on Comparison of Received Signal Strength Indicator. In: Proceedings of the IEEE International Conference on Mobile Ad-hoc and Sensor Systems. (October 2004) 516-518

5. Liu, B.C., Lin, K.H., Wu, J.C.: Analysis of Hyperbolic and Circular Positioning Algorithms Using Stationary Signal-Strength-Difference Measurements in Wireless Communications. IEEE Transactions on Vehicular Technology 55(2) (March 2006) 499-509 
6. Liu, B.C., Lin, K.H.: Distance Difference Error Correction by Least Square for Stationary Signal-Strength-Difference-based Hyperbolic Location in Cellular Communications. IEEE Transactions on Vehicular Technology 57(1) (January 2008) 227-238

7. Bulusu, N., Heidemann, J., Estrin, D.: GPS-less Low-Cost Outdoor Localization for Very Small Devices. IEEE Personal Communications 7(5) (October 2000) 28-34

8. Blumenthal, J., Grossmann, R., Golatowski, F., Timmermann, D.: Weighted Centroid Localization in Zigbee-based Sensor Networks. In: Proceedings of the IEEE International Symposium on Intelligent Signal Processing (WISP). (October 2007) 1-6

9. LAN/MAN Standards Committee of the IEEE Computer Society: IEEE Standard for Information Technology - Telecommunications and Information Exchange Between Systems - Local and Metropolitan Area Networks - Specific Requirements - Part 15.4: Wireless Medium Access Control (MAC) and Physical Layer (PHY) Specifications for Low-Rate Wireless Personal Area Networks (WPANS) - Amendment 1: Add Alternate PHYs. IEEE Std 802.15.4a-2007 (August 2007)

10. Behnke, R., Timmermann, D.: AWCL: Adaptive Weighted Centroid Localization as an Efficient Improvement of Coarse Grained Localization. In: Proceedings of the 5th Workshop on Positioning, Navigation and Communication (WPNC). (March 2008) 243-250

11. Schuhmann, S., Herrmann, K., Rothermel, K., Blumenthal, J., Timmermann, D.: Improved Weighted Centroid Localization in Smart Ubiquitous Environments. In: Ubiquitous Intelligence and Computing: Proceedings of the 5th International Conference (UIC). Volume 5061 of Lecture Notes in Computer Science., Springer Berlin / Heidelberg (2008) 20-34

12. Orooji, M., Abolhassani, B.: New Method for Estimation of Mobile Location Based on Signal Attenuation and Hata Model Signal Prediction. In: Proceedings of the IEEE 27th Annual Conference of the Engineering in Medicine and Biology Society. (September 2005) 6025-6028

13. Muir, A.: Automatic Sales Forecasting. The Computer Journal 1(3) (1958) 113-116

14. Roberts, S.W.: Control Chart Tests Based on Geometric Moving Averages. Technometrics 1(3) (August 1959) 239-250

15. Rappaport, T.S.: Wireless Communications: Principles and Practice. Second edn. PrenticeHall, New Jersey (2002)

16. Liechty, L.C.: Path Loss Measurements and Model Analysis of a $2.4 \mathrm{GHz}$ Wireless Network in an Outdoor Environment. Master's thesis, Georgia Institute of Technology (August 2007)

17. Liechty, L.C., Reifsnider, E., Durgin, G.: Developing the Best $2.4 \mathrm{GHz}$ Propagation Model from Active Network Measurements. In: Proceedings of the 66th IEEE Vehicular Technology Conference. (September 2007) 894-896

18. ASTM International: Standard Specification for Telecommunications and Information Exchange Between Roadside and Vehicle Systems - 5 GHz Band Dedicated Short Range Communications (DSRC) Medium Access Control (MAC) and Physical Layer (PHY) Specifications. ASTM E2213-03 (September 2003)

19. Durgin, G., Rappaport, T.S., Hao, X.: Measurements and Models for Radio Path Loss and Penetration Loss In and Around Homes and Trees at 5.85 GHz. IEEE Transactions on Communications 46(11) (November 1998) 1484-1496

20. Schwengler, T., Gilbert, M.: Propagation Models at $5.8 \mathrm{GHz}$ - Path Loss \& Building Penetration. In: Proceedings of the IEEE Radio and Wireless Conference (RANCOM). (September 2000) 119-124

21. Laurendeau, C., Barbeau, M.: Wireless Network-based Relative Span Weighted Localization of Uncooperative Nodes. Technical Report TR-09-04, School of Computer Science, Carleton University (March 2009) 